\title{
A characterization of the Hermitian variety in finite 3-dimensional projective spaces
}

\author{
Vito Napolitano* \\ Dipartimento di Matematica e Fisica \\ Seconda Università degli Studi di Napoli \\ Caserta, ITALY. \\ vito.napolitano@unina2.it
}

Submitted: May 30, 2013; Accepted: Jan 24, 2015; Published: Feb 9, 2015

Mathematics Subject Classifications: 05B25, 51E20

\begin{abstract}
A combinatorial characterization of a non-singular Hermitian variety of the finite 3-dimensional projective space via its intersection numbers with respect to lines and planes is given.
\end{abstract}

Keywords: projective space; intersection number; hermitian variety

\section{Introduction}

Ever since the celebrated theorem of B. Segre [10] on $(q+1)$-arcs of PG(2,q), $q$ odd, people have tried to characterize classical objects of finite projective geometry via their combinatorial properties. Intersection numbers with respect to the subspaces of a given dimension of the $d$-dimensional projective space $\operatorname{PG}(d, q)$ of order $q$ have been used frequently for this purpose.

Let us fix some notation. Let $\mathbb{P}=\mathrm{PG}(d, q)$ and let $m_{1}, \ldots, m_{s}$ be $s$ integers such that $0 \leqslant m_{1}<\ldots<m_{s}$. For any integer $h, 1 \leqslant h \leqslant r-1$, let $\mathcal{P}_{h}$ denote the family of all $h$-dimensional subspaces of $\mathbb{P}$. A subset $\mathcal{K}$ of points of $\mathbb{P}$ has class $\left[m_{1}, \ldots, m_{s}\right]_{h}$ for some $s$ if $|\mathcal{K} \cap \pi| \in\left\{m_{1}, \ldots, m_{s}\right\}$ for any $\pi \in \mathcal{P}_{h}$. Moreover, if for every $m_{j} \in\left\{m_{1}, \ldots, m_{s}\right\}$ there is at least one subspace $\pi \in \mathcal{P}_{h}$ such that $|\mathcal{K} \cap \pi|=m_{j}$ the set $\mathcal{K}$ is of type $\left(m_{1}, \ldots, m_{s}\right)_{h}$. In this case, the non-negative integers $m_{1}, \ldots, m_{s}$ are the intersection numbers of $\mathcal{K}$ (with respect to $\mathcal{P}_{h}$ ). If $h=1$ or $h=2$, we speak of the line-type or plane-type, respectively.

A wide literature is devoted to the theory of sets of a given type, some of which is listed in the references. The interest in studying such sets, in particular for the case of two

*Supported by G.N.S.A.G.A. of INdAM. 
intersection numbers with respect to hyperplanes is motivated in part by their connection with coding theory (cf e.g. [4, 7, 15]).

A non-singular hermitian variety of $\operatorname{PG}\left(3, q^{2}\right)$ has size $\left(q^{3}+1\right)\left(q^{2}+1\right)[6]$. Any line intersects the variety in either 1 , or $q+1$, or $q^{2}+1$ points. A plane intersects in either $q^{3}+1$ or $q^{3}+q^{2}+1$ points.

In this paper, we will give a new combinatorial characterization of a non-singular hermitian variety of the finite 3 -dimensional projective space. This result generalizes an earlier result due to Schillewaert and Thas [8].

Theorem I Let $\mathcal{K}$ be a set of $k=m(q+1)$ points of $\mathrm{PG}(3, q)$, for some integer $m$. Assume that the line type of $\mathcal{K}$ is $(1, s+1, q+1)_{1}$, for some integer $s$ with $1 \leqslant s \leqslant q-1$. Assume further that the plane type is $(m, h)_{2}$ for some integer $h$, then $q=s^{2}$ and $\mathcal{K}$ is a hermitian surface of $\mathrm{PG}\left(3, s^{2}\right)$.

As is costumary in the literature, a $j$-line is a line intersecting $\mathcal{K}$ in exactly $j$ points. A $j$-plane is a plane intersecting $\mathcal{K}$ in exactly $j$ points. For simplicity, a 1 -line is called a tangent line.

\subsection{Basic equations of $k$-sets of type $(m, h)_{2}$ in $\mathrm{PG}(3, q)$}

De Finis [3] studied combinatorial properties of sets in $\operatorname{PG}(3, q)$. Assume that $\mathcal{K}$ is a set of $k$ points in $\operatorname{PG}(3, q)$ with plane type $(m, h)_{2}$, for two distinct integers $m$ and $h$. Then $k$ is a solution to the equation

$$
k^{2}(q+1)-k\left[(h+m)\left(q^{2}+q+1\right)-q^{2}\right]+h m(q+1)\left(q^{2}+1\right)=0 .
$$

As a preparation for the arguments in the remainder of this paper, we outline a proof of this result:

Let $c_{j}$ be the number of $j$-planes of $\mathcal{K}$. Double counting gives

$$
\begin{gathered}
c_{m}+c_{h}=\left(q^{2}+1\right)(q+1) \\
m c_{m}+h c_{h}=k\left(q^{2}+q+1\right) \\
m(m-1) c_{m}+h(h-1) c_{h}=k(k-1)(q+1)
\end{gathered}
$$

from which the quadratic equation for $k$ follows.

We observe the following:

Observation 1: If $h=m+q$ the quadratic equation (1) has the two solutions

$$
k=m(q+1) \text { and } k=\frac{\left(q^{2}+1\right)(q+m)}{q+1} .
$$

Observation 2: If $k=m(q+1)$ the quadratic equation (1) implies that $h=m+q$.

The present paper is concerned with only the first case of the two cases occuring in (1) when $h=m+q$. 
If $\mathcal{K}$ is a $k$-set of $\operatorname{PG}(3, q)$ with intersection numbers $m$ and $m+q$ with respect to planes, it follows from the basic equations above that $k=m(q+1)$ or $k=\left(q^{2}+1\right)(q+m) /(q+1)$. Hence, in $\mathrm{PG}\left(3, q^{2}\right)$, from $m=q^{3}+1$ it follows that $k=\left(q^{3}+1\right)\left(q^{2}+1\right)=m\left(q^{2}+1\right)$ and so Theorem I generalizes Theorem 4.1 of [8].

Let us end this section with some remarks. In $\mathrm{PG}(3, q)$ a set of line type $(n)_{1}$ is either the empty set or the whole space, and a set of line type $(m, q+1)_{1}$ is either $\operatorname{PG}(3, q)$ less a point $(m=q)$ or a plane $(m=1)$ (cf e.g. [13]). If $\mathcal{K}$ is a set of points of $\mathrm{PG}(3, q)$ of line type $(1, n)_{1}$ then by results in [13] $n=q+1$ and so $\mathcal{K}$ is a plane. Thus, to study subsets of $\mathrm{PG}(3, q)$ of class $[1, a, q+1]_{1}$ means to study sets of line type $(1, a, q+1)_{1}$.

\section{The proof}

Throughout this section, $\mathcal{K}$ is a set of points of $\mathrm{PG}(3, q)$ of size $k=m(q+1)$ with line type $(1, s+1, q+1)_{1}$ and with plane type $(m, h)_{2}, s \geqslant 1$. It follows from Observation 2 that $h=m+q$.

Lemma 1. If $\ell$ is a $(q+1)$-secant line then all the planes containing $\ell$ are $h$-planes.

Proof. Let $\alpha$ denote the number of $m$-planes through $\ell$. Counting $k$ via the planes on $\ell$ gives

$$
m(q+1)=k=q+1+\alpha(m-q-1)+(q+1-\alpha)(m-1)
$$

So

$$
m(q+1)=m(q+1)-\alpha q
$$

from which it follows that $\alpha=0$.

Corollary 2. There are no $(q+1)$-lines contained in $m$-planes.

Lemma 3. Any m-plane contains at least one tangent line.

Proof. Recall that every line intersects $\mathcal{K}$ in either $1, s+1$, or $q+1$ points for some $s$ with $1 \leqslant s \leqslant q-1$. Assume that there is an $m$-plane $\pi$ containing no tangent line. If $\pi$ contains a $(q+1)$-line, then by Lemma $1, \pi$ is an $h$-plane. This contradiction shows that all lines of $\pi$ are $(s+1)$-secant lines. Let $p$ be a point of $\mathcal{K} \cap \pi$. Counting $m$ via the lines on $p$ gives $m=1+(q+1) s=s q+s+1$. Counting the incident point-line pairs $(p, \ell)$, $p \in \pi \cap \mathcal{K}$ gives

$$
(s q+s+1)(q+1)=m(q+1)=\left(q^{2}+q+1\right)(s+1)
$$

and so $s=q$, which is a contradiction. Hence any $m$-plane contains at least one tangent line.

Lemma 4. $m \leqslant s q+1$. 
Proof. Let $\pi$ be an $m$-plane and $\ell$ be a line of $\pi$ tangent to $\mathcal{K} \cap \pi$ at the point $p$. Let $x$ be the number of tangent lines on $p$. Counting points of $\mathcal{K} \cap \pi$ via the lines on $p$ gives

$$
m=1+(q+1-x) s,
$$

since $x \geqslant 1$ it follows that $m \leqslant s q+1$.

Lemma 5. $m=s q+1$.

Proof. Assume that $m \leqslant s q$. Thus, $h=m+q \leqslant(s+1) q$. Assume that there exists a $h$-plane $\pi$ containing two or more $(q+1)$-secant lines. Let $x$ be a point of $\pi$ not in $\mathcal{K}$. All lines of $\pi$ on $x$, except possibly for one, intersect $\mathcal{K} \cap \pi$ in at least two and hence in at least $s+1$ points, so $h \geqslant 1+q \cdot(s+1)$, a contradiction. Hence, every $h$-plane contains at most one $(q+1)$-line. Let $\ell$ be a $(q+1)$-line, let $\pi$ be an $h$-plane through $\ell$ and let $x$ be a point of $\mathcal{K} \cap \pi$ outside $\ell$. Counting points of $\mathcal{K} \cap \pi$ via the lines of $\pi$ passing through $x$ gives $h=1+(q+1) s=s q+s+1$.

Let $\alpha$ be an $m$-plane and $\mathcal{K}^{\prime}=\mathcal{K} \cap \alpha$. The set $\mathcal{K}^{\prime}$ has size $m$ and is of line type $(1, s+1)_{1}$. Let $p$ be a point of $\mathcal{K}^{\prime}$, and $x$ be the number of tangent lines on $p$. Counting points of $\mathcal{K} \cap \alpha$ via the lines on $p$ gives $m=1+(q+1-x) s$, so

$$
s q+s+1-q=h-q=m=1+s q+s-x s
$$

that is,

$$
x=\frac{q}{s} .
$$

Let $b_{1}$ and $b_{s+1}$ denote the number of tangent lines and $(s+1)$-lines of $\alpha$, respectively. Then

$$
m \frac{q}{s}=b_{1}
$$

that is, $m q=s b_{1}$.

On the other hand, $b_{1}+b_{s+1}=q^{2}+q+1$ and $b_{1}+(s+1) b_{s+1}=m(q+1)$. These last two equations imply that

$$
s b_{1}=(s+1)\left(q^{2}+q+1\right)-m(q+1) .
$$

Therefore,

$$
m q=(s+1)\left(q^{2}+q+1\right)-m(q+1)
$$

and so

$$
2 q+1=\frac{(s+1)\left(q^{2}+q+1\right)}{m}=\frac{(s+1)\left(q^{2}+q+1\right)}{s q+s+1-q} .
$$

Thus,

$$
\begin{aligned}
& 2 q=\frac{s q^{2}+q^{2}+2 q}{s q+s+1-q} \\
& s q^{2}+2 s q-3 q^{2}=0
\end{aligned}
$$


from which it follows that $s=2$ and $q=4$. Therefore, $m=7, h=11$ and $k=35$. Let $b_{i}, i \in\{1,3,5\}$, denote the number of $i$-lines of $\mathrm{P} G(3,4)$. The usual counting arguments give:

$$
\left\{\begin{array}{cccc}
b_{1}+b_{3}+b_{5}= & \left(q^{2}+1\right)\left(q^{2}+q+1\right)=357 \\
b_{1}+3 b_{3}+5 b_{5}= & k \cdot\left(q^{2}+q+1\right)=735 \\
6 b_{3}+10 b_{5}= & k(k-1)=1190
\end{array},\right.
$$

so, subtracting the first equation from the second one and dividing the third equation above by 2 give

$$
b_{3}+2 b_{5}=189 \text { and } 3 b_{3}+5 b_{5}=595
$$

which is a contradiction.

Lemma 6. $q=s^{2}$.

Proof. Let $\pi$ be an $m$-plane, $p$ be a point of $\pi \cap \mathcal{K}$, and let $x$ be the number of tangent lines of $\pi$ passing through $p$. Counting points of $\mathcal{K} \cap \pi$ via the lines on $p$ gives

$$
s q+1=m=1+(q+1-x) s
$$

and so $x=1$. Thus, every point of $\pi$ in $\mathcal{K}$ is on exactly one tangent line. So the numbers $b_{1}$ of tangents and $b_{s+1}$ of $(s+1)$-lines of $\pi$ are $b_{1}=m=s q+1$ and $b_{s+1}=q^{2}+q+1-m$, respectively. Counting the incident point-line pairs $(p, \ell)$ of $\pi, p \in \mathcal{K}, \ell$ a $(s+1)$-secant line gives

$$
(s q+1) q=(s+1) b_{s+1},
$$

from which it follows that

$$
b_{s+1}=q^{2}-\frac{q(q-1)}{s+1}
$$

Hence,

$$
\begin{gathered}
q^{2}-\frac{q(q-1)}{s+1}=b_{s+1}=q^{2}+q+1-s q-1 \\
(s-1) q=\frac{q(q-1)}{s+1} \\
s^{2}-1=q-1 .
\end{gathered}
$$

Thus, $m=s^{3}+1, h=s^{3}+s^{2}+1, k=\left(s^{3}+1\right)\left(s^{2}+1\right)$ and each line intersects $\mathcal{K}$ in 1 , $s+1$ or $s^{2}+1$ points. Hence, $\mathcal{K}$ is a $k_{n, 3, q}$ set in $\operatorname{PG}(3, q)$ (cf $\left.[6]\right)$ with $k=(q \sqrt{q}+1)(q+1)$, $n=s+1$ and $q=s^{2}$. So, since $\mathcal{K}$ contains no plane, $n \neq 1, q$, any point of $\mathcal{K}$ is on at least one $(s+1)$-secant line and for $q=4$ no 13 -plane contains three 5 -lines forming a triangle and $k=45$, it follows by the results in ([6], Section 19.5 Theorem 19.5.13) that $\mathcal{K}$ is a Hermitian variety of $\operatorname{PG}\left(3, s^{2}\right)$.

\section{Acknowledgements}

The author would like to thank the anonymous Referee for a careful reading of the original manuscript and making suggestions which have improved the presentation. 


\section{References}

[1] L. Berardi, T. Masini. On sets of type $(m, n)_{r-1}$ in $\mathrm{PG}(r, q)$. Discrete Math., 309:1629-1636, 2009.

[2] A. Bichara. Sui $k$-insiemi di $S_{3, q}$ di tipo $((n-1) q+1, n q+1)_{2}$. Rend. Acc.Naz. Lincei, 628(60):480-488, 1977.

[3] M. de Finis. On $k$-sets of type $(m, n)$ in $\mathrm{PG}(3, q)$ with respect to planes. Ars Combin., 21:119-136, 1986.

[4] R. Calderbank, W. M. Kantor. The geometry of two-weight codes. Bull. London Math. Soc., 18:97-122, 1986.

[5] O. Ferri. Le calotte a due caratteri rispetto ai piani in uno spazio di Galois $S_{r, q}$. Riv. Mat. Univ. Parma, IV (6):55-63, 1980.

[6] J. W. P. Hirschfeld. Finite projective spaces of three dimensions. Oxford University Press, 1985.

[7] V. Napolitano, D. Olanda, Sets of type $(3, h)_{2}$ in PG(3,q). Atti Accad. Naz. Lincei Cl. Sci. Fis. Mat. Natur. Rend. Lincei Mat. Appl., 22:1-9, 2012.

[8] J. Schillewaert, J. A. Thas, Characterizations of Hermitian varieties by intersection numbers. Des. Codes Cryptogr., 50:41-60, 2009.

[9] J. Schillewaert, A Characterization of quadrics by intersection numbers. Des. Codes Cryptogr., 47:165-175, 2008.

[10] B. Segre, Ovals in a finite projective plane. Canad. J. Math., 7:414-416, 1955.

[11] G. Tallini, Some new results on sets of type $(m, n)$ in projective planes. J. Geom., 29:191-199, 1987.

[12] M. Tallini Scafati, Caratterizzazione grafica delle forme hermitiane di un $S_{r, q}$. Rend. Mat. Roma, 26:273-303, 1967.

[13] M. Tallini Scafati, Sui $k$-insiemi di uno spazio di Galois $S_{r, q}$ a due soli caratteri nella dimensione d. Rend. Acc.Naz. Lincei, 8(60):782-788, 1976.

[14] J. A. Thas, A combinatorial problem. Geom. Dedicata, 1(2):236-240, 1973.

[15] J. H. Van Lint, A. Schrijver, Constructions of strongly regular graphs, two-weight codes and partial geometries by finite fields. Combinatorica, 1:63-73, 1981. 


\section{Corrigendum added March 292019}

A step in the proof of Theorem I is Lemma 5, which by contradiction gives that $m=s q+1$. In the proof of Lemma 5 , the author first proves that if $m \leqslant s q$ then $\mathcal{K}$ is a hypothetical set of points of $\operatorname{PG}(3,4)$ of size 35 , intersected by any plane in $m=7$ or $h=11$ points and by every line in 1, 3 or 5 points. Then using the usual incidence equations, with one of them written in a wrong way, he proves that such a set cannot exist, believing wrongly to have obtained the final contradiction. Indeed, in the last part of the proof of Lemma 5 the following system of linear equations and argument are used.

Let $b_{i}, i \in\{1,3,5\}$, denote the number of $i$-lines ${ }^{1}$. The usual point-line incidence counting arguments give:

$$
\left\{\begin{array}{cccc}
b_{1}+b_{3}+\quad b_{5} & = & \left(q^{2}+1\right)\left(q^{2}+q+1\right)=357 \\
b_{1}+3 b_{3}+5 b_{5} & = & k \cdot\left(q^{2}+q+1\right)=735 \\
6 b_{3}+10 b_{5} & = & k(k-1)=1190
\end{array}\right.
$$

so, subtracting the first equation from the second one and dividing the third equation above by 2 give

$$
b_{3}+2 b_{5}=189 \text { and } 3 b_{3}+5 b_{5}=595
$$

which is a contradiction.

The mistake is that the third equation should be $6 b_{3}+20 b_{5}=1190$, and so the above argument does not work.

Now, let us consider the previous system with the correct third equation. Thus, $b_{5}=7$ and $b_{1}=b_{3}=175$.

Let $\ell$ and $\ell^{\prime}$ two 5 -lines and assume that they intersect each other in a point $p$. The plane $\pi$ containing $\ell$ and $\ell^{\prime}$ is an $h$-plane since has at least 9 points. Let $x$ be a point of $\ell \backslash\{p\}$, the lines on $x$ in $\pi$ and different from $\ell$ have at least three points in $\mathcal{K} \cap \pi$ and so $\mathcal{K}$ intersects $\pi$ in at least $5+4 \cdot 2=13>11=h$ points, a contradiction.

Hence the seven 5 -lines are pairwise skew and so they form a partial spread of PG $(3,4)$. These lines partition the set of points of $\mathcal{K}$, and since $\mathcal{K}$ is of line type $(1,3,5)_{1}$ it follows that there is no line skew to all of them. Therefore such a partial spread is maximal. But this is a contradiction, since the number of lines of a maximal partial spread in $\operatorname{PG}(3, q)$ is at least $2 q(\mathrm{cf}[2,1])$, and so in this case it should be $7 \geqslant 2 q=8$. Hence, the case $s=2$, $q=4, m=7, h=11$ and $k=35$ cannot occur and so Lemma 5 is valid.

Let us end, by recalling that in [3] Hirschfeld and Hubaut gave the complete list of sets of line-type $(1,3,5)_{1}$ in $\mathrm{PG}(3,4)$ and that list contains no set of size 35 , so one may obtain the validity of Lemma 5 also via that result.

Additional correction to text: line 7 , Section 1 : ' $1 \leqslant h \leqslant r-1$ ' should be ' $1 \leqslant h \leqslant d-1$ '.

\section{Acknowledgements}

I would like to thank Professor Michel Lavrauw and his student Çiğdem Çelik who let me know of the error in the above incidence equation.

\footnotetext{
${ }^{1}$ An $i$-line (or $i$-plane) is a line (plane) intersecting $\mathcal{K}$ in exactly $i$-points.
} 


\section{References}

[1] A. A. Bruen, J. A. Thas, Partial Speads, Packings and Hermitian manifold in PG(3,q). Math. Z. 151 (1976), 207-214.

[2] D. G. Glynn, A lower bound for maximal partial spreads in PG(3, q). Ars Combinatoria 13 (1982), 39-40.

[3] J. W. P. Hirschfeld, X. Hubaut, Sets of Even Type in PG(3,4), alias the Binary $(85,24)$ Projective Geometry Code. J. Combin. Theory A 29 (1980), 101-112 